\title{
Roth spots in chronic myelogenous leukemia
}

\author{
Ronak K. Kapadia MD, Jeffrey H. Steeves MD
}



Figure 1: Retinal photographs of the right eye of a 39-year-old woman presenting with a two-month history of visual complaints, headache and night sweats. (A) Dilated examination of the posterior segment showed multiple white-centred hemorrhages (1 mm) consistent with Roth spots (arrows). Aspiration of the patient's bone marrow confirmed a diagnosis of chronic myelogenous leukemia. (B) After five weeks, the lesions are mostly resolved following treatment.

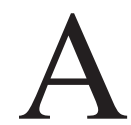

39-year-old woman presented with a history of headache and visual complaints including bright flashes of light, white "moth-like" areas and black spots throughout the field of vision in her right eye that had been ongoing for one to two months. In the days leading up to her presentation, her symptoms had progressed to involve both fields of vision. In addition to the changes in her vision, the patient described having night sweats during the same period.

A dilated eye examination showed multiple 1$\mathrm{mm}$ hemorrhages with white centres scattered throughout the retinas of both eyes (Figure 1A), consistent with Roth spots. Our patient's white blood cell count was 316 (normal range 3.5$11.0 \times 10^{9} / \mathrm{L}$ ), and aspiration of the patient's bone marrow confirmed a diagnosis of chronic myelogenous leukemia.

The patient was given imatinib, after which her visual symptoms, headaches and night sweats abated; after five weeks, her retinal lesions had decreased substantially in both number and severity (Figure 1B).

Roth spots were once believed to be pathognomonic of subacute bacterial endocarditis. The white-centred retinal hemorrhages are now seen as a nonspecific sign caused by the rupture of retinal capillaries, followed by the aggregation of fibrin and platelets. ${ }^{1}$ The range of clinical conditions associated with Roth spots is varied and includes subacute bacterial endocarditis, sepsis, toxoplasmosis, HIV, leukemia, diabetes, hypertension, vasculitis and traumatic brain injuries in infants..$^{1-3}$ Finding Roth spots should lead clinicians to consider investigations appropriate to the clinical context.

\section{References}

1. Ling R, James B. White centered retinal haemorrhages (Roth spots). Postgrad Med J 1998;74:581-2.

2. Falcone PM, Larrison WI. Roth spots seen on ophthalmoscopy: diseases with which they may be associated. Conn Med 1995;59: 271-3.

3. Reddy SC, Jackson N. Retinopathy in acute leukaemia at initial diagnosis: correlation of fundus lesions and haematological parameters. Acta Ophthalmol Scand 2004;82:81-5.
Competing interests: None declared.

This article has been peer reviewed.

Affiliations: From the Department of Clinical Neurosciences (Kapadia), University of Calgary, Calgary, Alta.; and the Department of Ophthalmology (Steeves), Saint John Regional Hospital, Saint John, NB

Acknowledgement: We thank Jeff Herc for preparing the retinal photographs.

Correspondence to: ronak.kapadia @ albertahealthservices.ca

CMAJ 2011. DOI:10.1503 /cmaj.100561 